$\xi=-1$

\title{
Design and development of information using keyword-element relationship graph - a critical study
}

\author{
M. V. B. T. Santhi ${ }^{1}$, S. Sagar Imambi ${ }^{1}$, J. Yamini Devi ${ }^{2}$, D. Tejaswani ${ }^{3}$ \\ ${ }^{1}$ Associate Professor, Computer Science and Engineering Department, Koneru Lakshmaiah Education Foundation, Guntur, A.P, India \\ ${ }^{2}$ Assistant Professor, Computer Science and Engineering Department, Koneru Lakshmaiah Education Foundation, Guntur, A.P, India \\ ${ }^{3}$ Student, Computer science and Engineering Department, Koneru Lakshmaiah Education Foundation, Guntur, A.P, India \\ *Corresponding author E-mail: simambi@kluniversity.in
}

\begin{abstract}
These days there is a gigantic accumulation of information and content reports are present. So there is fundamental for comprehension the vast content gathering of data. To comprehend the huge content accumulation of information is currently more simple by finding a string might be a characteristic perfect model for looking for associated information sources on the net. We have a tendency to propose to course fundamental words essentially to uncommon sources to reduce the high cost of unequivocal word look questions over all sources. We have a tendency to use a watchword component relationship dynamic that negligibly addresses associations inside of the center of catchphrases and consequently the information components indicating them. We would indicate controlling extensively serves to help the execution of definitive word search for, while not about its result quality.
\end{abstract}

Keywords: Graph-Structured Data; Keyword Search; Keyword Query Mapping Keyword Search; RDF Sources Resource Description Framework.

\section{Introduction}

Web is no additionally assembling of scholarly records yet it is similarly a web of interlinked know-how sources. One venture that to an uncommon degree gives to this development is Connecting Open know-how. Through doing this wander, a lot of legacy data had been changed over to RDF, connected with uncommon sources, and possible as associated know-how. Associated learning join many sources, which contains billions of RDF triples, that are related by a huge amount of associations. The place different associations may likewise be developed up; the ones each now and again allocated are equivalent as associations, that mean two RDF resources address the equivalent certifiable article. One such example of associated learning on the web is spoken to in Fig. 1.

ProductName: Manali

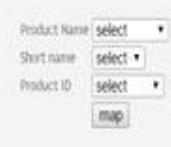

ProductlD: man7800

Shortameman

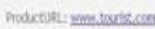

Product Description:Manali is a good place

Product Type:Tourist

ProductName: Goa

Fig: 1: Mapping Elements.
This is troublesome for web clients to make utilization of this webdata by method for organized questions utilizing dialects like SPARQL or SQL. Accordingly, catchphrase seek has ended up being intuitive [2]. As contrasting to organized inquiries, no learning of the construction, question dialect, the basic information that are required.

In database research, courses of action had been proposed, given a catchphrase query, get well likely the most related prepared outcomes or earnestly, pick without a doubt the most related databases.[7]. Be that as it will, these approaches are took into consideration single-supply. They are not surely related to the online of linked data, the outcomes are not bound by method for a lone supply however on the other hand could fuse only a couple connected comprehension sources. As separating to the supply determination quandary, which is concentrating on handling the premier sources, the primary trouble here is to enlist presumably the most monster blend of sources. The principal objective is to get steering arranges, that can be used to determine results from special sources.

To this problem, we provide the following work:

We inform to find the problem concerning catchphrase request controlling for watchword appear to be over a titanic number of prepared and associated know-how sources. Coordinating watchwords just to associated sources can reduce the over the top cost of seeking down arranged result that navigate a considerable amount of sources. The work that is acquainted inside the paper talks with the most critical practice to address this hassle where present work uses catchphrase associations (KR) which can be assembled solely for single databases. The connections between watchwords and in addition those between information components which are built for the whole accumulation of connected sources, and afterward assembled as components of a conservative outline called the setlevel catchphrase component relationship diagram (KERG). Condensing these connections is crucial for tending to the versatility 
necessity of the Connected Information web situation. IR-style positioning has been proposed with a specific end goal to incorporate pertinence at the level of catchphrases. To adapt up to the expanded catchphrase vagueness in the web setting, we make utilization of multilevel significance model, where the components to be considered are watchwords, comparing sets of substances, connections between components of same level, and between connections between components of various levels

\section{Materials and methods}

\subsection{Searching for a keyword}

Current work can be sorted into two major courses: There are arrangement gather methods completed in gentle of prime off-therack databases[1].. A watchword request is good to go by method for mapping catchphrases to additional items of the database. By then, by making utilization of outline, enormous join plans are gotten, that are then used to wind up distinctly an individual from ("buddy") the prepared watchword segments to casing contender strategies conversing with conceivable result to the catchphrase address. Improvement freethinker approaches work clearly on the learning. Equipped outcomes are prepared with the guide of looking at the concealed comprehension outline. Statute idea is to recognize structures inside the know-how called Steiner trees, associate catchphrase frill. Phenomenal diverse sorts of counts have been proposed for the powerful examination of watchword record things over skill graphs, that maybe sweeping.

Outlines are bidirectional chase and detail programming . Starting late, a structure alluded to as Kite extends design based strategies to find candidate techniques inside the multisource setting. It makes utilization of outline planning methods to search out joins inside the center of sources and uses constitution disclosure frameworks to notice remote key joins across over sources. These are likewise in view of precomputed connections, Hermes that makes an interpretation of catchphrases to organized questions. Be that as it may, tests have been performed just for a little number of sources as such.

Kite has considered just the setting where "the quantity of databases that can be managed is up to the tens". The inquiry space bit by bit increments, furthermore, the quantity of potential results might increment exponentially with the quantity of sources and connections between them. However, a large portion of the outcomes might be redundant particularly when they are not significant to the client. Presumably the most answer for watchword inquiry discarding to have the capacity to direct can address these clutters pointless sources and engaging customers to choose blends that more conceivable incorporate huge outcomes. For the coordinating issue, there is no convincing method of reasoning to decide result getting exact parts at the understanding level, however to focus on the more coarse-grained level of sources.[5].

\subsection{Mapping the keyword data}

The information used for the trials are drawn from learning sets orchestrated the Billion Triple Challenge1(BTC).BTC know-how were crept from huge derivation sites amidst February/walk 2009. BTC information were half into chunks of $10 \mathrm{M}$ declarations each. All the additional information, and bits of knowledge are made open at http://vmlion25. deri.ie/index.html. the learning we tend to utilized for the examination are the irregularity that might be found at http://vmlion25.deri.ie/btc-2009-little.nq.gz. This bit of information contains endless sources. Various of them contain less than 3000 RDF triples. Proposed Framework shows estimations for every classification and a couple representation sources.

1) Data Pre-processing

Amidst the record building technique, we checked the quantity of watchword affiliations, i.e., all courses of action of charm expressions which are joined over a most best separation operations That is to take after the M-KS mannequin, which will get each two fold association between convincing words. As specified, EKERG enlarges G-KS to the charm expression managing situation [3]. We counted the amount of segment degree key expression stage affiliations (E-KERs) to catch this outline. Along these lines, we remember the amount of relationship in KERG (KERs).

These numbers had been suggested the total data and selfgoverningly for every last subcategory. At different dmax, follows the amount of KRs versus E-KERs versus KERs for the whole information. These showcase the amount of KERs, the stockpiling size required for the viewing at KERG archives, and the best open door for building these records for information units of unmistakable characterizations. None the less, we saw that these result were not a considerable measure reliant on the data measurement. That is, the amount of KERs, the scope of the rundown moreover setting up times didn't specifically join with the amount of triples contained in the data units. There have been events the place appropriately little data units done extensive KERGs. For cost, we can see that events for classes of bigger in estimation have been more prominent than these of negligible estimation. In any case, even as order 2 was yet again than a hundred and fifty rate better in size, the refinement in once-over building time to gathering 3 used to be under 5 rate. On this, we can see that at dmax $1 / 44$, the amount of KERs and the report measurement of collection 2 have been much more diminutive than these of advancement 3 .

\section{2) Queries}

Our essential goals of the evaluation square \{measure to substantiate the legitimacy and measure the relevance of the made polar word managing game plans. For a preparation to be significant, the required inquiry got the chance to convey answers. Further, interesting questions amid this setting zone unit those that combine results from different sources. we have a tendency to asked pros WHO were familiar with the BTC information set to exhibit unequivocal word request that return required results, on board portrayals of the arranged Information needs. Inside and out, we've thirty enchantment word request; everything about incorporate more than 2 information sources. One example given by individuals is "Rudi AIFB ISWC2008," furthermore the associated depiction is "Discover the associations between Prof Rudi Studer, the AIFB Organization furthermore the ISWC'2008 gathering." the information sources containing down reactions to the present request territory unit uni-karlsruhe.de and semanticweb.org.[6]. The catchphrases of the underlying twenty inquiries zone unit in contestable in arranged Framework.

\section{Existing system}

There square measure mapping outlined philosophies dead in lightweight of prime of off-the-peg databases. An unequivocal word request is revised by mapping catchphrases to parts of the information (called watchword segments). Around then, using the arrangement, honest to goodness be a piece of groupings square measure deduced, that is then used to hitch ("unite") the prepared unequivocal word parts to structure assumed cheerful frameworks visiting with possible results to the maxim request.

Outline nonreligious individual systems work particularly on the information. Sorted out results square measure enlisted by examination the natural information chart. The objective is to get structures inside of the information known as Steiner trees (Steiner charts beat all), that interface aphorism parts. Contrasting sorts of counts are made arrangements for the ability full examination of crucial word question, which may be extensive. Cases square measure bifacial request and part programming Existing take an attempt at definitive word search for relies on upon a component level - model (i.e., information diagrams) to enroll maxim question results.

\subsection{Disadvantages of existing system}

- The potential results might increment exponentially with the quantity of sources and connections existing. Yet, a large 
portion of the outcomes might be a bit much particularly when they are not pertinent to the client.

- In the directing issue, we have to register results catching particular components at the information level.

- Routing catchphrases give back the whole source, which might possibly be the applicable sources.

\section{Proposed system}

We advocate to path catchphrases simply to exceptional sources to lessen the high improvement of nurturing urgent word get ask for from various core interests. We have a tendency to propose a one of a kind method for enrolling top-k controlling courses of action in lightweight of their possibilities to contain results for a given key inquiry of word. We have a tendency to use an unequivocal word component relationship characterize that insignificantly addresses associations inside of the center of catchphrases furthermore the information parts voice correspondence them.

A structure rating instrument is gotten capable for strategy the significance of controlling arrangements in lightweight of rankings on the measure of huge words, understanding fixings, angle sets, and sub outlines so one can talk to these materials.

We have a tendency to propose to take a gander at the issue of enchantment word request directing for maxim look over an inside and out assortment of sorted out and coupled data sources.

3) Advantages of proposed system

- Steering watchwords to only relevant sources can chop down the high price of looking down sorted out outcomes that cross more than a couple sources.

- The steerage executions, which are made, can be used to process comes about because of incalculable sources.

\section{Results}

1) Create admin.

2) Login admin and add product details with product name, short name and product id.

3) User can search the keywords and searching process will be done by mapping the data selected by the user.

4) Results will be displayed.

5) The user can also view the raking of the keyword.

Discovering the distinctive linguistics elucidations of a magic word inquiry could be a combinatorial issue which might be explained by an intensive identification of the varied ways in which mappings may be associated with info structures. Each decisive word could be a specific concerning the element of hobby. The determination is displayed within the info as a social table, a characteristic, or Associate in Nursing estimation of an attribute. A setup could be a mapping capability that depicts a determination for each question magic word as way as info terms.

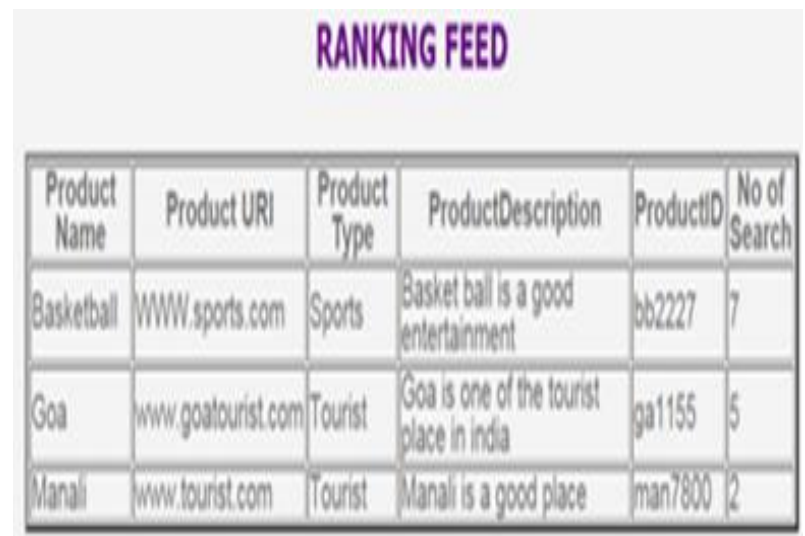

Fig: 2: Ranking Display.

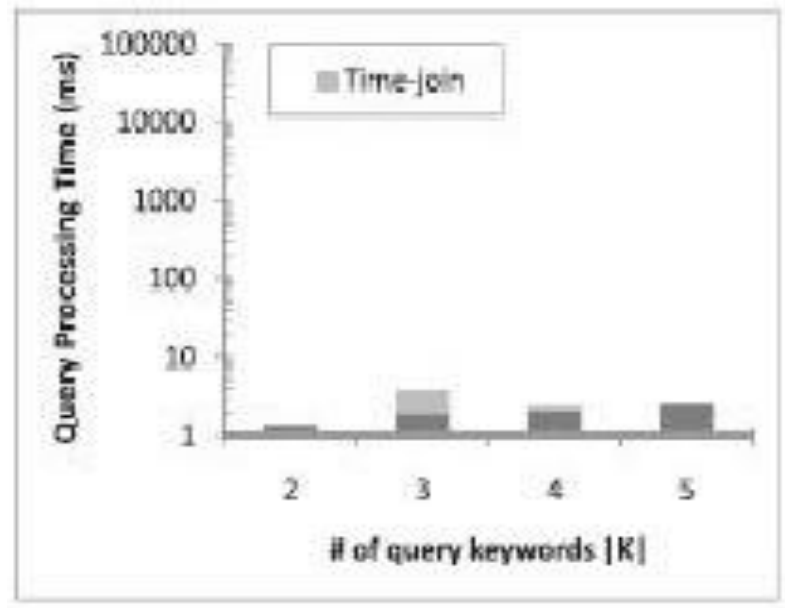

Fig 3: Performance Evaluation of Query.

By considering significance marking the productivity of inquiry framework is computed. We have a tendency to do this examination for set of catchphrases and determined score in light-weight of their significance and gave positioned list things. This kind of seeking strategy is a lot of solid and productive inquiry system that's a lot of liable to produce applicable results than typical inquiries. Our significance score examination results demonstrate that the projected hunt routines tremendously enhance the effectiveness of positioned catchword obtain.

\section{Conclusion}

A multi-record content mapping in light of keyword element relationship structure is exhibited in this work. Tests are conveyed utilizing something like four hubs in clustering system for an extensive content accumulation and the rundown execution parameters pressure proportion, maintenance proportion and calculation timings are assessed for an extensive content accumulation. It is additionally indicated tentatively that MapReduce system gives better versatility also, decreased time many-sided quality while considering expansive number of content archives for outline. Three conceivable instances of outlining the different reports are too concentrated relatively. It is demonstrated that viable rundown is performed when both grouping and semantic comparability considered. Considering semantic closeness gives better maintenance proportion, ROUGE and pyramid scores for outline.

\section{References}

[1] Elmasri Navrate, Data base Management System, Pearson Education (2010), pp: 69-72.

[2] Rob \& Coronel, Data base Systems design, Implementation, and Management, Thomson, 5th Edition.

[3] B. Yu, G. Li, K.R. Sollins, and A.K.H. Tung (2007), Effective Keyword- Based Selection of Relational Databases, Proc. ACM SIGMOD Conf., and pp. 139-150.

[4] Serge Abiteboul, Foundations of Databases, Richard Hull and Victor Vianu.

[5] Prachi M.Karale,Natikar S.B(2014), A Survey on Keyword Query Routing,'Int'l Journal of Advance Research in Computer Science and Engineering, Vol.2, No.2

[6] Mrs. Suwarna Gothane, Srujana. P (2014), Approaches for Keyword Query Routing", Int. Journal of Engineering Research and Applications, Vol.4, No.10.

[7] V. Hristidis, L. Gravano, and Y. Papakonstantinou (2003), Efficient IR-.Style Keyword Search over Relational Databases, Proc. 29th Int'l Conf. Very LargData Bases (VLDB), pp. 850-861. 